\title{
Psoas muscle size as a frailty measure for open and transcatheter aortic valve replacement
}

\author{
Raghavendra Paknikar, BS, ${ }^{\text {a,d }}$ Jeffrey Friedman, BS, ${ }^{\mathrm{d}}$ David Cron, BS, ${ }^{\mathrm{d}}$ G. Michael Deeb, MD, \\ Stanley Chetcuti, MD, ${ }^{b}$ P. Michael Grossman, MD, ${ }^{\mathrm{b}}$ Stewart Wang, MD, PhD, ${ }^{c, d}$ Michael Englesbe, MD, ${ }^{c, d}$ \\ and Himanshu J. Patel, MD ${ }^{\text {a,d }}$
}

\begin{abstract}
Objective: To evaluate the use of sarcopenia as a frailty assessment tool for patients with aortic stenosis undergoing surgical aortic valve replacement (SAVR) or transcatheter aortic valve replacement (TAVR).

Methods: The study cohort comprised 295 patients who underwent either SAVR $(\mathrm{n}=156)$ or TAVR $(\mathrm{n}=139)$. The mean preoperative Society of Thoracic Surgeons mortality risk score was $4.7 \%$. Preoperative computed tomography (CT) scans were used to calculate gender-standardized total psoas area (TPA), as a validated measure of sarcopenia.
\end{abstract}

Results: For the entire cohort, independent predictors of a composite measure of 30-day death, stroke, renal failure, prolonged ventilation, and deep wound infection included preoperative STS major morbidity and mortality risk score (odds ratio $[\mathrm{OR}], 91.1 ; P=.02$ ) and TPA (OR, $0.5 ; P=.024)$. Two-year survival was $85.7 \%$ in patients with sarcopenia, compared with $93.8 \%$ in patients without sarcopenia $(P=.02)$. Independent predictors of late survival included TPA (hazard ratio, $0.47 ; P=.02)$. Male sex (OR, 0.52; $P=.04)$ and TPA (OR, 0.6; $P=.001)$ were predictive of high resource utilization. A separate analysis by treatment group found that TPA predicted high resource utilization after SAVR (OR, 0.4; $P<.001)$, but not after TAVR $(P=.66)$.

Conclusions: CT scan-derived measurement of TPA as an objective frailty assessment tool predicts early morbidity and mortality, high resource utilization, and late survival after treatment for aortic stenosis. The correlation observed between sarcopenia and resource utilization after SAVR versus TAVR suggests that this simple and reproducible risk assessment tool also may help identify those patients who will derive optimal benefit from catheter-based therapy. ( $\mathrm{J}$ Thorac Cardiovasc Surg 2016;151:745-51)

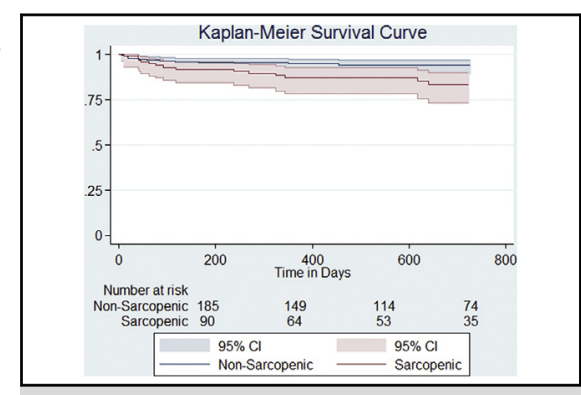

Reduction of psoas muscle area (sarcopenia) is an important predictor of late mortality.

\section{Central Message}

Sarcopenia is an objective frailty assessment tool for predicting outcomes following either open or transcatheter aortic valve replacement.

\section{Perspective}

Reduction of psoas muscle area (sarcopenia) has been used as an objective tool to assess frailty. This study suggested that it may be predictive of adverse outcomes after open surgical and transcatheter aortic valve replacement. This simple and reproducible test easily obtained with routine preoperative imaging may help further define the group of patients who can derive optimal benefit from a catheterbased approach.

See Editorial Commentary page 752

\footnotetext{
From the Departments of ${ }^{\mathrm{a}}$ Cardiac Surgery, ${ }^{\mathrm{b}}$ Medicine, and ${ }^{\mathrm{c}}$ Surgery and the ${ }^{\mathrm{d}}$ Morphomic Analysis Group, University of Michigan Medical Center, Ann Arbor, Mich.

This work was generously supported by the David Hamilton Fund and the Phil Jenkins Breakthrough Fund in Cardiac Surgery.

Read at the 95th Annual Meeting of The American Association for Thoracic Surgery, Seattle, Washington, April 25-29, 2015.

Received for publication May 10, 2015; revisions received Oct 20, 2015; accepted for publication Nov 2, 2015.

Address for reprints: Himanshu J. Patel, MD, Department of Cardiac Surgery, CVC Rm 5144, 1500 E Medical Center Dr, SPC 5864, Ann Arbor, MI 48109-5864 (E-mail: hjpatel@med.umich.edu).

$0022-5223 / \$ 36.00$

Copyright (C) 2016 by The American Association for Thoracic Surgery

http://dx.doi.org/10.1016/j.jtcvs.2015.11.022
}

The recent advent of transcatheter aortic valve replacement (TAVR) has altered the paradigm of care for those patients traditionally considered at high or extreme risk for conventional surgical aortic valve replacement (SAVR). ${ }^{1-3}$ Although risk stratification algorithms have been used to evaluate perioperative outcomes, these scores typically evaluate comorbidities rather than assess frailty. ${ }^{4}$ Despite a dramatic improvement in survival with TAVR, the recently reported PARTNER B trial found a high 1-year mortality for the entire cohort regardless of chosen treatment algorithm. ${ }^{1}$ With the emergence of TAVR, 


$$
\begin{aligned}
& \text { Abbreviations and Acronyms } \\
& \begin{aligned}
\text { CABG } & =\text { coronary artery bypass grafting surgery } \\
\text { CI } & =\text { confidence interval } \\
\text { CT } & =\text { computed tomography } \\
\text { HR } & =\text { hazard ratio } \\
\text { OR } & =\text { odds ratio } \\
\text { SAVR } & =\text { surgical aortic valve replacement } \\
\text { STS } & =\text { Society of Thoracic Surgeons } \\
\text { TAVR } & =\text { transcatheter aortic valve replacement } \\
\text { TPA } & =\text { total psoas area }
\end{aligned}
\end{aligned}
$$

difficulties with frailty assessment have risen to the forefront to avoid implementation of TAVR in futile patients (the so-called "cohort C"). ${ }^{5}$

Previous work from our group has demonstrated the utility of analytic morphomics in describing the observed relationship between loss of core muscle mass (sarcopenia) and patient frailty. ${ }^{6-8}$ In the present study, we used readily available cross-sectional imaging to assess core muscle size to aid risk stratification after major general and vascular surgery. Here we describe its application in patients undergoing either SAVR or TAVR as a novel risk assessment tool to aid therapeutic decision making. Our study hypothesis was that the presence of sarcopenia could suggest selection of the less-invasive TAVR for patients who would otherwise struggle to recover from SAVR owing to underlying frailty.

\section{METHODS}

This retrospective study was approved by the University of Michigan's Institutional Review Board. All patients undergoing SAVR with or without coronary artery bypass grafting (CABG) or TAVR between 2011 and 2013 were identified $(\mathrm{n}=710)$. After excluding patients with lack of adequate preoperative (within 90 days) computed tomography (CT) imaging of the L1 to L4 vertebral levels without distortion from orthopedic hardware, a final cohort of 295 patients (mean age, 74.4 years) constituted the study group. In this cohort, $156(52.9 \%)$ underwent SAVR with or without CABG, and the remaining $139(47.1 \%)$ underwent TAVR. Clinical data were collected from our Society of Thoracic Surgeons (STS) local database, and the STS risk calculator was applied to all patients to determine an overall risk profile. The mean mortality risk score for the cohort was $4.66 \%$.

Using the available imaging data, psoas major core muscle size as an indicator of sarcopenia was obtained as described previously. ${ }^{6-8}$ In brief, analytic morphomic processing was completed in a semiautomated fashion using algorithms programmed in MATLAB version 13.0 (MathWorks, Natick, Mass). Scans were first anatomically indexed by mapping out individual vertebral levels. The psoas muscles were outlined at the inferior border of the fourth lumbar vertebrae (Figure 1). The areas of the left and right psoas muscles were summed to compute the total psoas area (TPA). For analysis, TPA was standardized by gender to control for differences in body habitus. TPA values were interpreted as the number of standard deviations (SDs) from the mean value of their gender within this study cohort.

The primary outcome measure of this study was late mortality, derived from use of clinically available data from patient charts and by interrogation of the Social Security Death Index. High resource utilization, a secondary outcome measure, was a dichotomous composite measure consisting of length of stay (>14 days), readmission within 30 days, and/ or postoperative extended intensive care unit stay ( $>7$ days). Patients

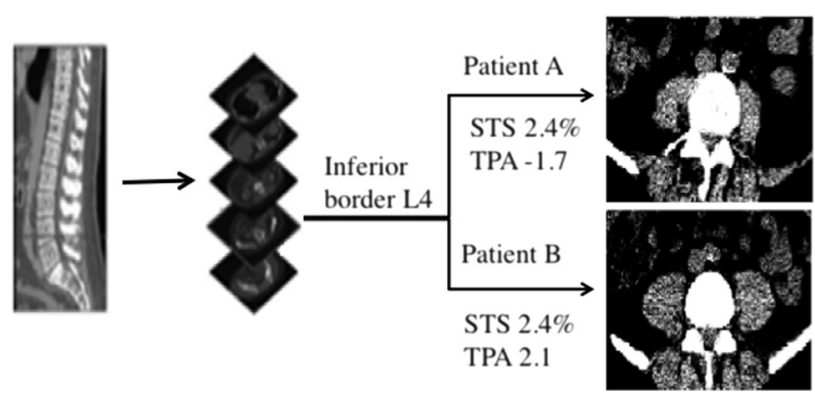

FIGURE 1. Schematic outlining the process for generating total psoas area. Cross-sectional imaging identifies the inferior border of L4, and the total psoas major muscle area is obtained at this level. This example identifies 2 patients with similar STS mortality risk scores (patient A, $2.35 \%$; patient $\mathrm{B}, 2.43 \%$ ). As is readily evident, patient $\mathrm{A}$ has a reduced total psoas area, consistent with sarcopenia (1.72 SD below the gender mean). In contrast, patient B has a robust total psoas area (2.08 SD above the gender mean. In this case, the patient with sarcopenia required readmission within 30 days (high resource utilizer), unlike patient B, who had no such adverse outcome. STS, Society of Thoracic Surgeons; $T P A$, total psoas area.

were considered to be high resource utilizing if they had any of the foregoing 3 attributes. The final secondary outcome measure was a composite metric of major early morbidity or mortality, and consisted of perioperative occurrence of renal failure (STS definition version 2.73), stroke, reoperation during index hospitalization, prolonged ventilation $>24$ hours, and 30-day mortality.

\section{Statistical Analysis}

Variables were described as mean $\pm \mathrm{SD}$ and number and proportion where appropriate. Univariate analysis was performed with either the unpaired Student $t$ test or $\chi^{2}$ test. Multivariable analysis was conducted using binary logistic regression for dichotomous variables or Cox regression analysis for continuous variables. Variables included in the backward stepwise selection process included those identified as significant on univariate analysis, at a $P$ value $<.10$.

Propensity score adjustment by logistic regression analysis was performed to account for selection bias preferentially shunting higher-risk patients toward the TAVR group. The type of operative procedure was the dependent variable, and clinical variables affecting the probability of selecting patients for TAVR evaluated in the propensity score calculation included the presence of severe chronic lung disease, infectious endocarditis, previous coronary intervention or bypass surgery, congestive heart failure, serum albumin, presence of liver disease, STS risk scores for mortality and combined mortality and major morbidity, gender, nonelective status, presence of tricuspid insufficiency, hypertension, and cardiac presentation. The propensity score thus developed was then included in all original multivariable analyses evaluating outcomes for the entire cohort.

\section{RESULTS}

Demographic and comorbidity data are presented in Table 1. Compared with the patients undergoing SAVR, those undergoing TAVR were older (79.0 years vs 70.4 years; $P<.001)$ and had a higher STS mortality risk score $(6.5 \%$ vs $3.0 \% ; P<.001)$. The burden of almost all evaluated comorbidities was higher in the TAVR group, as expected. Again as expected, mean TPA was significantly different between males and females $\left(2659 \pm 711 \mathrm{~mm}^{2}\right.$ vs 
TABLE 1. Demographic and comorbidity data

\begin{tabular}{|c|c|c|c|}
\hline Characteristic & $\begin{array}{l}\text { SAVR group } \\
(\mathbf{n}=\mathbf{1 5 6})\end{array}$ & $\begin{array}{l}\text { TAVR group } \\
(\mathbf{n}=139)\end{array}$ & Univariate $P$ value \\
\hline Age, year, mean \pm SD & $70.40 \pm 13.76$ & $78.96 \pm 8.92$ & $<.001$ \\
\hline Body mass index, mean $\pm \mathrm{SD}$ & $30.57 \pm 6.01$ & $29.58 \pm 7.14$ & .1985 \\
\hline $\mathrm{STS}$ mortality risk score, mean $\pm \mathrm{SD}$ & $3.04 \pm 3.28$ & $6.48 \pm 4.52$ & $<.001$ \\
\hline STS major morbidity and mortality risk score, mean \pm SD & $18.22 \pm 10.25$ & $29.15 \pm 11.22$ & $<.001$ \\
\hline Gender-standardized TPA, mean \pm SD & $0.25 \pm 1.05$ & $-0.29 \pm 0.85$ & $<.001$ \\
\hline Female sex, n $(\%)$ & $49(31)$ & $53(38)$ & .226 \\
\hline Diabetes, $\mathrm{n}(\%)$ & $41(26)$ & $61(44)$ & .002 \\
\hline Hypertension, $\mathrm{n}(\%)$ & $118(76)$ & $139(80)$ & .386 \\
\hline Peripheral vascular disease, $\mathrm{n}(\%)$ & $17(11)$ & $42(30)$ & $<.001$ \\
\hline Cerebrovascular disease, $\mathrm{n}(\%)$ & $20(13)$ & $46(33)$ & $<.001$ \\
\hline Previous cerebrovascular accident, $\mathrm{n}(\%)$ & $7(4)$ & $17(12)$ & .015 \\
\hline Previous CABG, n $(\%)$ & $16(10)$ & $60(43)$ & $<.001$ \\
\hline Previous valve, n (\%) & $14(9)$ & $15(11)$ & .601 \\
\hline Previous percutaneous coronary intervention, $\mathrm{n}(\%)$ & $31(20)$ & $53(38)$ & .001 \\
\hline Myocardial infarction within 21 days, $\mathrm{n}(\%)$ & $3(2)$ & $2(1)$ & .748 \\
\hline Left main disease, $\mathrm{n}(\%)$ & $7(4)$ & $17(12)$ & .014 \\
\hline Current and/or recent smoker, $\mathrm{n}(\%)$ & $14(9)$ & $5(4)$ & .060 \\
\hline Infectious endocarditis, $\mathrm{n}(\%)$ & $8(5)$ & $1(1)$ & .028 \\
\hline Liver disease, $\mathrm{n}(\%)$ & $5(3)$ & $6(4)$ & .615 \\
\hline Immunosuppressive treatment, $\mathrm{n}(\%)$ & $6(4)$ & $17(12)$ & .007 \\
\hline Reoperation, n (\%) & $32(21)$ & $73(53)$ & $<.001$ \\
\hline Urgent status, n (\%) & $25(16)$ & $14(10)$ & .132 \\
\hline Severe chronic lung disease, $\mathrm{n}(\%)$ & $3(2)$ & $17(12)$ & .001 \\
\hline New York Heart Association class 4, n (\%) & $4(3)$ & $25(18)$ & $<.001$ \\
\hline Ejection fraction, mean $\pm \mathrm{SD}$ & $60.41 \pm 12.02$ & $55.63 \pm 15.24$ & .003 \\
\hline Hematocrit, mean $\pm \mathrm{SD}$ & $38.23 \pm 5.75$ & $36.32 \pm 5.89$ & .005 \\
\hline Serum albumin, mean $\pm \mathrm{SD}$ & $4.24 \pm 0.42$ & $3.96 \pm 0.41$ & $<.001$ \\
\hline Serum creatinine, mean $\pm \mathrm{SD}$ & $1.13 \pm 0.85$ & $1.4 \pm 0.97$ & .013 \\
\hline
\end{tabular}

SAVR, Surgical aortic valve replacement; TAVR, transcatheter aortic valve replacement; $S D$, standard deviation; STS, Society of Thoracic Surgeons; TPA, total psoas area; $C A B G$, coronary artery bypass grafting.

$\left.1666 \pm 490 \mathrm{~mm}^{2} ; P<.001\right)$. Consequently, analysis of TPA was then standardized by gender. Gender-standardized TPA was lower in the TAVR group compared with the SAVR group $(-0.29 \pm 0.85$ below the mean vs $0.25 \pm 1.05$ above the mean; $P<.001)$. Within each treatment group, however, the lack of correlation between TPA and STS risk score (Figure 2) suggests the opportunity for a unique risk assessment tool for patients undergoing either therapeutic option.

Table 2 presents early and late results by treatment type. For the entire cohort, the composite measure of early poor
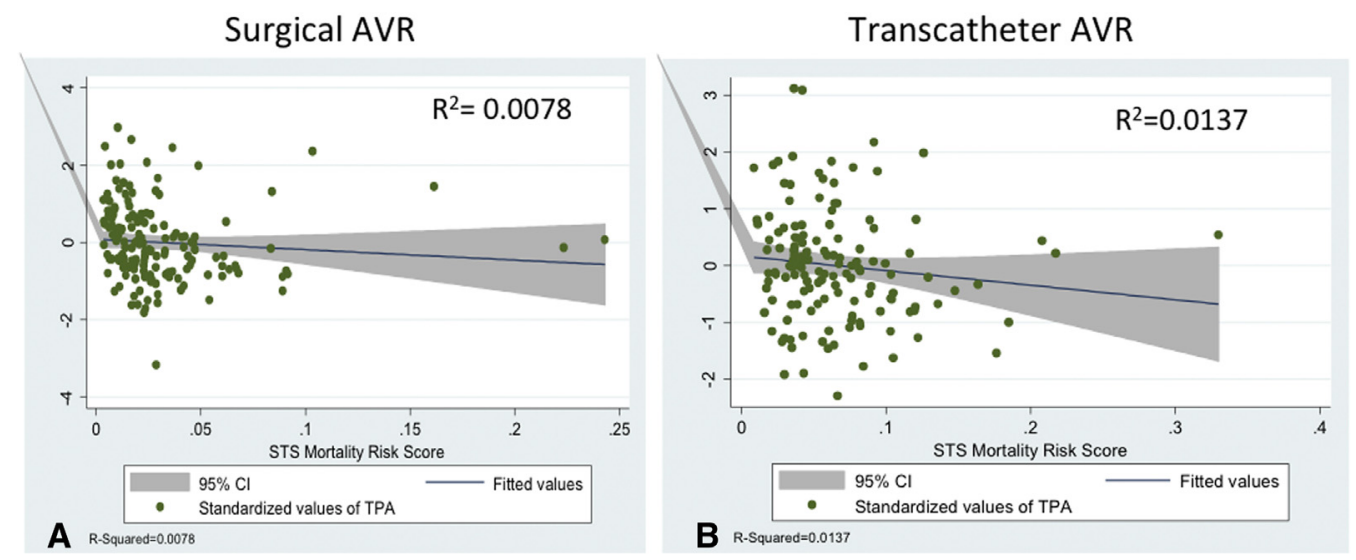

FIGURE 2. The lack of correlation between total psoas area ( $y$-axis) and STS mortality risk score ( $x$-axis) for the SAVR (A) and TAVR (B) groups. The lack of correlation in either cohort suggests an opportunity to develop a novel risk assessment domain in patients undergoing AVR. AVR, Surgical aortic valve replacement; STS, Society of Thoracic Surgeons; CI, confidence interval; TPA, total psoas area. 
TABLE 2. Early and late outcomes stratified by treatment

\begin{tabular}{|c|c|c|c|}
\hline Outcome variable & $\begin{array}{c}\text { SAVR } \\
\text { group } \\
(\mathrm{n}=156) \\
\end{array}$ & $\begin{array}{c}\text { TAVR } \\
\text { group } \\
(\mathbf{n}=\mathbf{1 3 9}) \\
\end{array}$ & $\begin{array}{c}\text { Univariate } \\
P \text { value } \\
\end{array}$ \\
\hline $\begin{array}{l}\text { Early composite poor outcome, } \\
\qquad \mathrm{n}(\%)^{*}\end{array}$ & $13(8)$ & $11(8)$ & 1.0 \\
\hline 30-d mortality, n (\%) & $0(0)$ & $5(4)$ & .022 \\
\hline Stroke, n (\%) & $2(1)$ & $6(4)$ & .154 \\
\hline Renal failure, $\mathrm{n}(\%) \dagger$ & $0(0)$ & $3(2)$ & .10 \\
\hline Deep wound infection, $\mathrm{n}(\%)$ & $6(4)$ & $1(0.7)$ & .125 \\
\hline $\begin{array}{l}\text { Prolonged ventilation, }>24 \text { hours, } \\
\text { n }(\%)\end{array}$ & $11(7)$ & $6(4)$ & .454 \\
\hline Late crude mortality, n (\%) & $5(3)$ & $21(15)$ & $<.001$ \\
\hline High resource utilization, n (\%) & $41(26)$ & $27(19)$ & 169 \\
\hline \multicolumn{4}{|c|}{$\begin{array}{l}\text { SAVR, Surgical aortic valve replacement; TAVR, transcatheter aortic valve replace- } \\
\text { ment. *Early composite poor outcome included } 30 \text {-day mortality, stroke, deep wound } \\
\text { infection, prolonged ventilation, and renal failure. } \dagger \text { Renal failure, as defined by STS } \\
\text { version } 2.73 \text {, requires increasing serum creatinine to } 3 \text { times the initial baseline value, } \\
\text { or serum creatinine } \geq 4 \mathrm{mg} / \mathrm{dL} \text { with an increase of at least } 0.5 \mathrm{mg} / \mathrm{dL} \text {, or new dialysis } \\
\text { requirement. }\end{array}$} \\
\hline
\end{tabular}

outcome occurred in 25 patients $(8.4 \%)$ and did not differ between the SAVR and TAVR groups $(P=1.0)$. Independent predictors of occurrence of early poor outcome included body mass index (odds ratio [OR], 1.1;
$P<.001)$, STS morbidity and mortality score (OR, 91.1; $P=.02)$, tobacco use (OR, $4.97 ; P=.031)$, or the presence of mitral stenosis (OR, 7.8; $P=.026)$. TPA was also predictive (OR, $0.52 ; P=.024)$, indicating that a single TPA measure of $1 \mathrm{SD}$ below the mean increased the occurrence of the early poor composite outcome by $48 \%$.

Late mortality in the entire cohort was observed in 26 patients $(8.8 \%)$. Sarcopenia was associated with a lower 2-year survival for the entire cohort $(85.7 \%$ for patients with sarcopenia vs $93.8 \%$ for those without sarcopenia; $P=.02, \log$-rank test) (Figure 3, A). A subsequent analysis was then performed separately for the SAVR and TAVR groups, and similar findings were obtained (Figure 3, B and $C$ ). By Cox regression analysis, independent predictors of late mortality included occurrence of myocardial infarction within 21 days (hazard ratio [HR], 9.2; $P=.009)$ and TPA (HR, 0.52; $P=.016)$.

Finally, 68 patients in the entire cohort $(23.1 \%)$ required high resource utilization, but rates were not significantly different between treatment arms $(P=.17)$. Independent predictors of higher resource utilization included male sex (OR, $0.5 ; P=.037$ ), presence of aortic insufficiency (OR, $0.42 ; P=.013)$, and TPA (OR, $0.56 ; P=.001)$. The
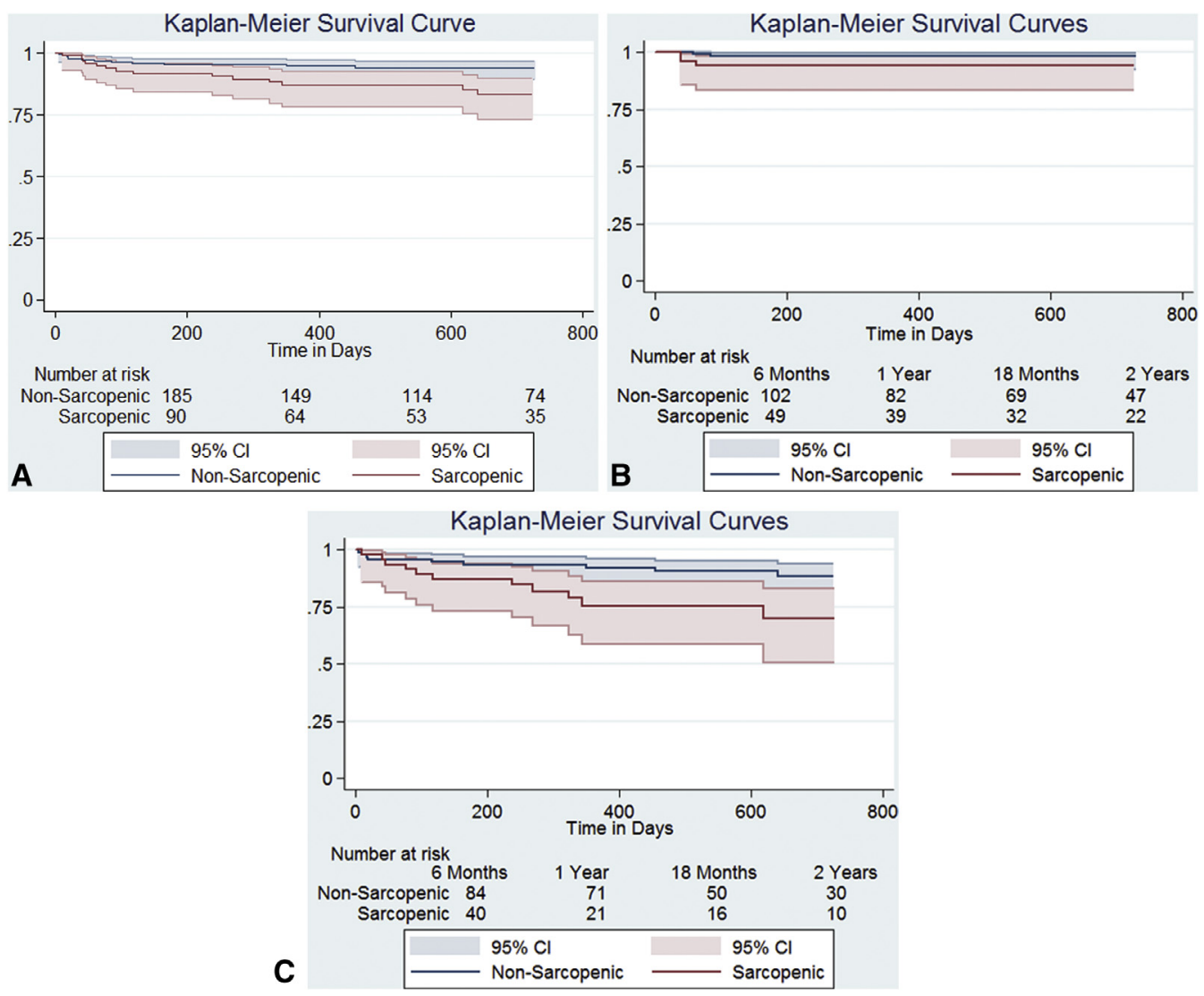

FIGURE 3. A, The entire cohort stratified according to the presence of sarcopenia (lowest-tertile TPA; 2 -year survival, $85.7 \%$ ) and absence of sarcopenia (2-year survival, $93.8 \% ; P=.02, \log$-rank test). B, The SAVR group stratified by sarcopenia status $(P=.019, \log$-rank test). C, The TAVR group stratified by sarcopenia status $(P=.026, \log$-rank test $)$. All curves include $95 \%$ confidence interval shading for each stratified group. $C I$, Confidence interval. 


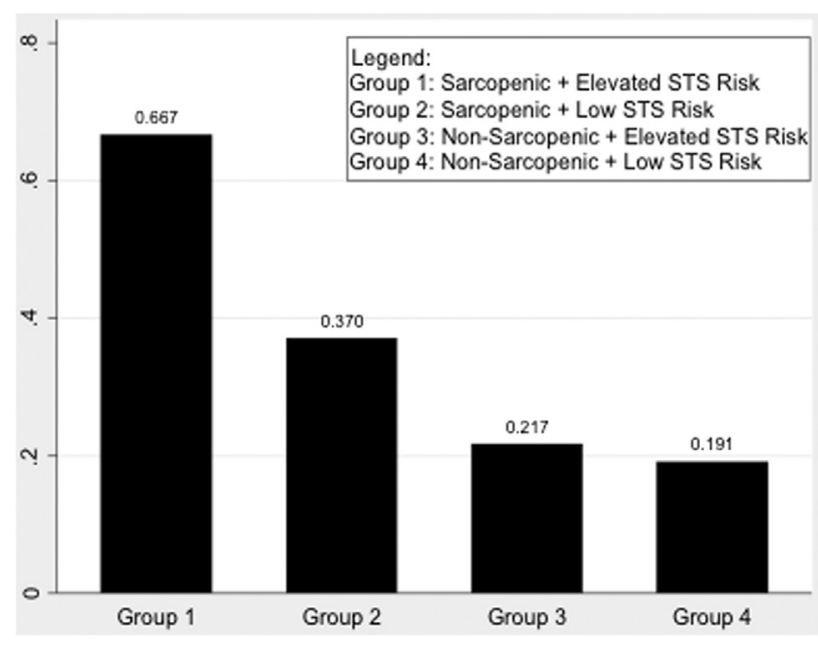

FIGURE 4. Mean high resource utilization rates stratified by TPA quartile and STS mortality risk score (either above or below 4\%). The risk of high resource utilization is greatest in patients with sarcopenia with intermediate or higher risk scores compared with patients without sarcopenia with low STS mortality risk scores $(66.7 \%$ vs $19.1 \%)$. STS, Society of Thoracic Surgeons.

analysis was then separated based on treatment algorithm. For the SAVR group, logistic regression identified TPA as an independent predictor of high resource utilization (OR, $0.41 ; P<.001)$. After adjusting for covariates, patients with a TPA below the gender mean had an adjusted mean high resource utilization rate of $35.2 \%$, whereas those with a TPA above the mean had a utilization rate of $16.3 \%$. Given the lack of correlation between the STS risk score, which primarily evaluates comorbidities, and sarcopenia (presumably evaluating frailty), we wished to analyze various combinations of these 2 scores on high resource utilization. Figure 4 present the results of this analysis, showing a high utilization rate of $66.7 \%$ for the group in the lowest quartile of TPA with an STS risk score $>4 \%$. In contrast, patients with an STS risk score $<4 \%$ and no evidence of sarcopenia had a utilization rate of $19.1 \%$. We then performed the analysis for the TAVR group and found that TPA was not a significant predictor of high resource utilization $(P=.662)$.

\section{DISCUSSION}

The recent success of catheter-based therapy for the treatment of aortic stenosis in patients at high or extreme risk has shifted the focus to concepts of frailty and futility in determining the optimal treatment for aortic stenosis in elderly patients. ${ }^{1-3,5,9-12}$ Although medical treatment portends poor survival and deteriorating quality of life, intervention in older debilitated patients requires the ability to determine that the therapy will positively alter the natural history of the disease..$^{9-15}$

Traditional objective risk assessment scores, such as the STS risk score, do not evaluate frailty, but rather stratify predominantly on the basis of comorbidities. ${ }^{4}$ In clinical practice, typical assessment of operative suitability in this patient group has included the "eyeball test," which is associated with significant variability and lack of objectivity. ${ }^{5}$ Newer, but cumbersome tools have been discussed, including gait speed, grip strength, and the recently proposed multidimensional geriatric assessment, but these are likely underused. ${ }^{9-12}$ Therefore, we undertook this study to evaluate a novel objective risk assessment tool that can be readily obtained from routine preoperative imaging studies in patients undergoing aortic valve replacement. Our previous work described the use of TPA as an important predictor of outcomes after various procedures. $^{6-8}$ For example, sarcopenia was identified as an important risk factor for 1-year mortality after abdominal aortic aneurysmectomy, but the impact on mortality was greatest early after surgery. ${ }^{7}$ Similarly, after esophagectomy, sarcopenia and pathological tumor stage had equal effects on disease-free interval and overall survival. ${ }^{8}$ Unlike other objective assessment tools, core muscle measurements are not subject to acute changes in body status (eg, arthritis or mood) or effort (eg, gait test) and can easily be standardized and evaluated.

Our results show that sarcopenia is an important independent predictor of adverse outcomes, such as early morbidity, late mortality, and high resource utilization in a cohort undergoing aortic valve repair in the TAVR era. The argument for considering evaluation for sarcopenia was strongest when evaluating predictors of high resource utilization. By combining this assessment with a global evaluation of comorbidities (STS risk score strata), we identified an even stronger predictor of high resource utilization. To mitigate the effects of treatment selection bias, we used propensity score adjustment for multivariate analysis. When this analysis was separated on the basis of treatment type, we identified its validity in predicting high resource utilization for those patients undergoing conventional SAVR. In contrast, the presence of sarcopenia was unable to predict high resource utilization after TAVR. This implication of superiority and suitability of TAVR in frail patients does not describe futility, however. Taken together, these results argue for the use of TPA as a measure of sarcopenia, along with the objective assessments of comorbidity and "heart team" evaluation, in determining optimal treatment choice. Alternatively, this tool may be important to help identify patients who may benefit from preoperative optimization, such as diet modification or physical therapy.

The most important next step is to attempt to identify differential effects of sarcopenia for early morbidity and late mortality in SAVR and TAVR. Although our study has identified the importance of sarcopenia in determining early and late mortality in the overall cohort, separation of analysis based on treatment group was not possible, given the relatively small sample size and lack of events 
in the SAVR group (data not shown). Another important step would be to compare TPA with conventional, simpler methods of frailty, such as grip strength, albumin, and others. We believe that this can be evaluated with data from recently performed multicenter randomized trials.

Limitations of this study include the use of the Social Security Death Index, in addition to a clinical chart review. The limited purging of entries after November 2011 likely would not have occurred selectively in patients with a particular TPA size, however, and also would not have increased the overall event rate, thereby making overcorrection bias unlikely. Other limitations of this study include the exclusion of many patients from the original cohort owing to inadequate imaging and, finally, that this particular frailty assessment tool does not evaluate cognitive and social considerations, which are important aspects of the frailty syndrome.

In conclusion, we suggest that a novel risk assessment tool to evaluate frailty is available from routine preoperative imaging studies. This estimate of frailty may provide physicians with an objective measure of risk assessment and aid in optimal selection of therapy for aortic stenosis in the transcatheter era.

\section{Conflict of Interest Statement}

Dr Chetcuti is a paid consultant for Medtronic. Dr Englesbe is an equity stakeholder in Prehab Tech and Prenovo. All other authors have nothing to disclose with regard to commercial support.

You can watch a Webcast of this AATS meeting presentation by going to: http://webcast.aats.org/2015/Video/ Monday/04-27-15_608_1645_Patel.mp4.

\section{References}

1. Leon MB, Smith CR, Mack MJ, Miller DC, Moses JW, Svensson LG, et al. Transcatheter aortic valve implantation for aortic stenosis in patients who cannot undergo surgery. N Engl J Med. 2010;363:1597-607.

2. Smith CR, Leon MB, Mack MJ, Miller DC, Moses JW, Svensson LG, et al. Transcatheter versus surgical aortic valve replacement in high risk patients. $N$ Engl J Med. 2011;364:2187-98.

3. Popma JJ, Adams DH, Reardon MJ, Yakubov SJ, Kleiman NS, Heimansohn D, et al. Transcatheter aortic valve replacement using a self-expanding bioprosthesis in patients with severe aortic stenosis at extreme risk for surgery. J Am Coll Cardiol. 2014;63:1972-81.

4. Thourani VH, Suri RM, Gunter RL, Sheng S, O'Brien SM, Ailawadi G, et al. Contemporary real-world outcomes of surgical aortic valve replacement in 141,905 low-risk, intermediate-risk, and high-risk patients. Ann Thorac Surg. 2015;99:55-61.

5. Mack MJ. Frailty and aortic valve disease. J Thorac Cardiovasc Surg. 2013; 145(3 Suppl):S7-10.

6. Englesbe MJ, Lee JS, He K, Fan L, Schaubel DE, Sheetz KH, et al. Analytic morphomics, core muscle size and surgical outcomes. Ann Surg. 2012;256: 255-61.

7. Lee JS, He K, Harbaugh CM, Schaubel DE, Sonnenday CJ, Wang SC, et al. Frailty, core muscle size, and mortality in patients undergoing open abdominal aortic aneurysm repair. J Vasc Surg. 2011;53:912-7.

8. Sheetz KH, Zhao L, Holcombe SA, Wang SC, Reddy RM, Lin J, et al. Decreased core muscle size is associated with worse patient survival following esophagectomy for cancer. Dis Esophagus. 2013;26:716-22.
9. Afilalo J, Eisenberg MJ, Morin JF, Bergman H, Monette J, Noiseux N, et al. Gait speed as an incremental predictor of mortality and major morbidity in elderly patients undergoing cardiac surgery. J Am Coll Cardiol. 2010;56: 1668-76.

10. Lee DH, Buth KJ, Martin BJ, Yip AM, Hirsch GM. Frail patients are at increased risk for mortality and prolonged institutional care after cardiac surgery. Circulation. 2010;121:973-8.

11. Sündermann S, Dademasch A, Praetorius J, Kempfert J, Dewey T, Falk V, et al. Comprehensive assessment of frailty for elderly high-risk patients undergoing cardiac surgery. Eur J Cardiothorac Surg. 2011;39:33-7.

12. Stortecky S, Schoenenberger AW, Moser A, Kalesan B, Jüni P, Carrel T, et al. Evaluation of multidimensional geriatric assessment as a predictor of mortality and cardiovascular events after transcatheter aortic valve implantation. JACC Cardiovasc Interv. 2012;5:489-96.

13. Fried LP, Tangen CM, Walston J, Newman AB, Hirsch C, Gottdiener J, et al. Frailty in older adults: evidence for a phenotype. J Gerontol A Biol Sci Med Sci. 2001;56:M146-56.

14. Fried TR, Mor V. Frailty and hospitalization of long-term stay nursing home residents. J Am Geriatr Soc. 1997;45:265-9.

15. Dewey TM. Frailty scores and the writing on the wall. JACC Cardiovasc Interv. 2012;5:497-8.

Key Words: aortic valve replacement, outcomes, transcatheter valve replacement

\section{Discussion}

Dr W. Szeto (Philadelphia, Pa). Himanshu, nicely done. Thank you for sending me the manuscript in advance, and I want to thank also the Association for the privilege of discussing this manuscript.

So your group has nicely demonstrated in the past that the psoas muscle area, is a nice way to identify poor outcome in general and vascular surgery, and you've translated this nicely into TAVR.

I think clearly from looking at your presentation that the surgical group and the TAVR group are very different, but nonetheless, I think this is interesting, and it does prove that for aortic valve disease patients, that sarcopenia is a nice potential new tool for us to determine if patients are either too sick or futile, and that's very important in today's medical care environment.

I have a couple questions for you. One, you've demonstrated nicely that the STS score absolutely had no correlation with psoas muscle area. In these same patient populations, did you also have the classic frailty assessment data, ie, the grip, albumin, walk test, and did you see any correlation between sarcopenia and frailty assessment? Or another way of looking at this is, did this tool allow you to identify another patient population that we would have otherwise missed with the traditional frailty assessment?

Dr Patel. Thank you, Wilson. That's a really good point. So we don't routinely perform these other frailty assessment tests in patients that undergo surgical aortic valve replacement, but I think that's why the study needs to be done in the big multicenter randomized studies for catheter-based therapy.

So I think it would be important as a next step to look at the PARTNER data or the CoreValve data because that does 
provide all of these things, and we would be able to get the psoas muscle area from those.

Dr Szeto. It should be fairly straightforward.

And question two, with this interesting data, how do we apply it? Is there a cutoff that you would then use and not recommend patients for surgical aortic valve replacement, and even more extreme, a cutoff that they're even too sick for TAVR? How do you use this data to help our patients? Thank you.

Dr Patel. Thank you, Wilson, for the thoughtful questions. We believe our study is a hypothesis-generating study that could be validated further by analysis of data from the large multicenter TAVR versus SAVR studies from the PARTNER and CoreValve study groups. This may validate our conclusions that suggest that certain patients with sarcopenia may be better treated with TAVR or it could also suggest that certain patients who may need SAVR may require optimization before surgery.

Dr G. Whitman (Baltimore, Md). Dr Patel, I'm not sure whether this is clear, but as with the pulmonary function studies that we were talking about with the previous paper, it would be hard, I think, to suggest that everybody about to undergo an aortic valve replacement should get a CT scan of the psoas muscle.

For those of us who don't know that much about frailty, is there a bedside test that correlates well with psoas muscle size, because if there is, then maybe we could assess frailty at the bedside. Otherwise, I cannot imagine getting CT scans on the 50 or 60,000 patients a year undergoing an aortic valve replacement.

Dr Patel. I can. I believe that, in the TAVR era, all patients who undergo SAVR should be evaluated with a CT scan if possible. This test may diagnose aneurysms, extensive calcification that may either alter cannulation sites or preclude one from SAVR among other things. At our center, we are very liberal in imaging a patient undergoing SAVR not only for these reasons but also because we believe in TAVR's bright future and look at all options, including TAVR for each patient, to ensure a complete assessment.

Dr Whitman. But is there a bedside test that correlates well with the results of the CT scan?

Dr Patel. Unfortunately, not that I know of.

Dr Whitman. Okay. With that, thank you very much. 